TRANSACTIONS OF THE

AMERICAN MATHEMATICAL SOCIETY

Volume 356, Number 12, Pages 4909-4929

S 0002-9947(04)03538-X

Article electronically published on February 4, 2004

\title{
CHARACTERIZATIONS OF REGULAR ALMOST PERIODICITY IN COMPACT MINIMAL ABELIAN FLOWS
}

\author{
ALICA MILLER AND JOSEPH ROSENBLATT
}

\begin{abstract}
Regular almost periodicity in compact minimal abelian flows was characterized for the case of discrete acting group by W. Gottschalk and G. Hedlund and for the case of 0-dimensional phase space by W. Gottschalk a few decades ago. In $1995 \mathrm{~J}$. Egawa gave characterizations for the case when the acting group is $\mathbb{R}$. We extend Egawa's results to the case of an arbitrary abelian acting group and a not necessarily metrizable phase space. We then show how our statements imply previously known characterizations in each of the three special cases and give various other applications (characterization of regularly almost periodic functions on arbitrary abelian topological groups, classification of uniformly regularly almost periodic compact minimal $\mathbb{Z}$ - and $\mathbb{R}$-flows, conditions equivalent with uniform regular almost periodicity, etc.).
\end{abstract}

\section{INTRODUCTION}

Regular almost periodicity was studied from the early days of Topological Dynamics. In the Gottschalk-Hedlund monograph [19] one of the chapters was dedicated to this notion. Among other results, they gave a characterization of uniform regular almost periodicity for the case when the acting group is discrete. They also gave various examples of pointwise and uniform regular almost periodicity in some natural flows that appear in symbolic dynamics.

In his paper [18], Gottschalk has further investigated regular almost periodicity. He studied the so-called trace relation of a compact minimal flow and gave several theorems expressing regular almost periodicity in terms of it. He also gave a characterization of uniform regular almost periodicity for compact minimal flows with a 0-dimensional phase space and an arbitrary acting group. Various examples of regular almost periodicity he gave came from symbolic dynamics.

Although various particular theorems about regular almost periodicity appeared in the literature for many years, no new characterization-type result had been found until 1995, when J. Egawa gave characterizations of both pointwise regular almost periodicity and regular almost periodicity of a point in compact minimal flows with the acting group $\mathbb{R}$. In his proofs Egawa essentially used the topological properties of the phase space implied by the assumption that the real line is continuously mapped (by any orbital map) onto a dense subset of it. He also assumed that the phase space is metrizable and, also essentially, used that assumption. There was no obvious connection between Egawa's and Gottschalk and Hedlund's results.

Received by the editors November 3, 2002 and, in revised form, June 19, 2003.

2000 Mathematics Subject Classification. Primary 37B05, 43A60; Secondary 43A40, 54H20.

Key words and phrases. Compact minimal flows, almost periodic, regularly almost periodic, almost automorphic, almost $1-1$, eigenvalues, 0-dimensional, Bohr compactification. 
Our goal is to extend Egawa's results to the case of an arbitrary abelian acting group and a not necessarily metrizable phase space.

Section 2 explains notations and introduces some preliminaries.

In Sections 3 and 4 we develop all the necessary tools that are used in the rest of the paper. Concretely, in Section 3 we show some statements about eigenvalues of almost periodic compact minimal flows, while in Section 4 we deal with some properties of flows, like regular almost periodicity, almost automorphicity, local almost periodicity, regional distality and establish some relations between them.

In Section 5 we introduce the "eigenvalue condition" and use it to formulate and prove our characterizations (Theorem 5.2 and Theorem 5.3). The statements are appropriate generalizations of Egawa's statements. Both statements are proved for arbitrary abelian toplogical acting groups and a not necessarily metrizable phase space.

In Section 6 we consider the case when the phase space is 0-dimensional. We first deduce from our Theorem 5.2 Gottschalk's statement which characterizes uniform regular almost periodicity in this case. In the remaining statements we are mainly concerned with various properties that are (weaker but) equivalent with uniform regular almost periodicity.

In Section 7 we end up with a characterization of regularly almost periodic functions on arbitrary abelian topological groups. In order to prove this characterization, we first develop the "harmonic analysis" of almost periodic functions on arbitrary abelian topological groups. This includes all the standard statements (the existence of mean value, the approximation theorem, the uniqueness theorem, the frequency module theorem), all given with very short proofs working for arbitrary abelian topological groups.

In Section 8 we consider the case when the acting group is discrete. We first deduce from our Theorem 5.2 Gottschalk and Hedlund's statement which characterizes uniform regular almost periodicity in this case. Then we consider some properties that are (weaker but) equivalent with uniform regular almost periodicity. Finally we give a classification of all uniformly regularly almost periodic compact minimal $\mathbb{Z}$-flows.

In Section 9 we first explain that in the case $T=\mathbb{R}$ our general context reduces to Egawa's context. Then we deduce from our Theorems 5.2 and 5.3 Egawa's characterization theorems. Since we did not start with the assumption that the phase space is metrizable, we were able to deduce that the phase space of every uniformly regularly almost periodic compact minimal $\mathbb{R}$-flow is necessarily metrizable (being a compact topological group with countably many continuous characters). We finish with a classification of all uniformly regularly almost periodic compact minimal $\mathbb{R}$-flows.

\section{Notations AND PRELIMinaries}

All topological spaces in this paper are assumed to be Hausdorff and all groups to be abelian. (Some of the preparatory statements that we use are also true for nonabelian groups, especially in Section 4 and the first two pages of Section 7 But most of the tools that we use, our main theorems and the applications are true only for abelian groups. So for the purpose of this paper it is enough to deal with abelian groups.) 
The topological space obtained when a set $X$ is equipped with the discrete topology is denoted by $X_{d}$. The letter $T$ will always denote a topological group. $C(T)$ denotes the set of continuous complex functions on $T . B U C_{u}(T)$ denotes the set of bounded uniformly continuous complex functions on $T$, equipped with the uniform norm. $\mathbb{T}$ will denote the topological group of complex numbers of module 1 . The continuous homomorphisms $\chi: T \rightarrow \mathbb{T}$ are called continuous characters of $T$. The set of all continuous characters of $T$ will be denoted by $\widehat{T}$. A subset $A$ of $T$ is syndetic if there exists a compact subset $K$ of $T$ such that $T=K+A$.

We use the theory of uniform spaces as it is presented in 21. The entourages (i.e., indices in [2, 12, 19, 28]) are denoted by small greek letters: $\alpha, \beta$, etc. If $X$ is a uniform space, its set of entourages is denoted by $\mathcal{U}_{X}$. If $\alpha \in \mathcal{U}_{X}$ and $x \in X$, then $\alpha[x]$ denotes the set of all $y \in X$ such that $(x, y) \in \alpha$.

A triple $\mathcal{X}=\langle T, X, \pi\rangle$ consisting of a topological group $T$, a topological space $X$ and a continuous action $\pi: T \times X \rightarrow X$ of $T$ on $X$ is called a flow on $X$. We write $t . x$ or $t x$ for $\pi(t, x) . \mathcal{X}$ is compact if $X$ is compact. For $x \in X$ we denote by $\pi^{x}: T \rightarrow X$ the orbital map $t \mapsto t . x$. For $t \in T$ we denote by $\pi_{t}$ the transition homeomorphism $x \mapsto t . x$. The flow $\mathcal{X}$ is trivial if $X$ is a singleton. If $U$ is a neighborhood of a point $x \in X$, we denote $D(x, U):=\{t \in T \mid t x \in U\}$.

When we have a $\mathbb{Z}$-flow on $X, \mathcal{X}=\langle\mathbb{Z}, X, \pi\rangle$, then the transition homeomorphism $h:=\pi_{1}$ completely defines the action: $\pi(n, x)=h^{n}(x)$. In that case we write $\mathcal{X}=\langle X, h\rangle$ for short.

A flow $\mathcal{X}_{S}=\left\langle S, X,\left.\pi\right|_{X \times S}\right\rangle(\langle S, X\rangle$ for short), where $S$ is a subgroup of $T$, is a restriction of the flow $\mathcal{X}=\langle T, X, \pi\rangle$. If a subset $Y$ of $X$ is invariant under the action of $T$, the canonical flow $\langle T, Y\rangle$ is a subflow of $\mathcal{X}$.

Let $\mathcal{Y}=\langle T, Y\rangle$ be another flow. A map $f: X \rightarrow Y$ is a morphism of flows if it is continuous and $f(t x)=t f(x)$ for all $t \in T$ and $x \in X$. If $f$ is surjective, $\mathcal{X}$ is an extension of $\mathcal{Y}$ and $\mathcal{Y}$ is a factor of $\mathcal{X}$. A bijective morphism of \{flows \{a flow on itself $\}$ is an $\{$ isomorphism $\}$ automorphism $\}$. A flow $\mathcal{X}$ is minimal if the orbit T.x of every point $x \in X$ is dense in $X$.

Let $\mathcal{X}=\langle T, X\rangle$ be a flow. A point $x \in X$ is almost periodic (in $\mathcal{X}$ ) if for every neighborhood $U$ of $x$ there is a syndetic subset $A$ of $T$ such that $A x \subset U$. If $\mathcal{X}$ is compact, $x$ is almost periodic if and only if $\overline{T x}$ is minimal. A flow $\mathcal{X}$ is pointwise almost periodic if every point $x \in X$ is almost periodic.

If $\mathcal{X}$ is a flow on a uniform space $\left(X, \mathcal{U}_{X}\right), \mathcal{X}$ is uniformly almost periodic if for every $\alpha \in \mathcal{U}_{X}$ there exists a syndetic set $A \subset T$ such that $A . x \subset \alpha[x]$ for every $x \in X$. In the case of compact flows, uniformly almost periodic flows are the same as (uniformly) equicontinuous flows (i.e., those flows for which the family of transition maps is (uniformly) equicontinuous) and are called simply almost periodic flows.

Let $\mathcal{X}$ be a flow on a compact (uniform) space $\left(X, \mathcal{U}_{X}\right)$. Two points $x, y \in X$ are proximal (to each other) if for every $\alpha \in \mathcal{U}_{X}$ there exists $t \in T$ such that $(t x, t y) \in \alpha$. The flow $\mathcal{X}$ is called proximal if all pairs of points are proximal. The relation " $x$ and $y$ are proximal" is called the proximal relation of $\mathcal{X}$ and is denoted by $P_{\mathcal{X}}$. We have $P_{\mathcal{X}}=\bigcap_{\alpha \in \mathcal{U}_{X}}$ T. $\alpha$. Two points $x, y \in X$ are distal (to each other) if they are not proximal. A point $x \in X$ is distal if it is distal to every point $y \in X \backslash\{x\}$; i.e., if $P_{\mathcal{X}}[x]=\{x\}$. The flow $\mathcal{X}$ is called distal if all its points are distal.

Two points $x, y \in X$ are regionally proximal (to each other) if for any neighborhood $U$ of $x$ and $V$ of $y$ and any $\alpha \in \mathcal{U}_{X}$ there are $x^{\prime} \in U, y^{\prime} \in V$ and $t \in T$ 
such that $\left(t x^{\prime}, t y^{\prime}\right) \in \alpha$. The relation " $x$ and $y$ are regionally proximal" is called the regionally proximal relation of $\mathcal{X}$ and is denoted by $Q_{\mathcal{X}}$. We have $Q_{\mathcal{X}}=\bigcap_{\alpha \in \mathcal{U}_{X}} \overline{T . \alpha}$.

If $P_{\mathcal{X}}=Q_{\mathcal{X}}$, the proximal relation $P_{\mathcal{X}}$ is a closed invariant equivalence relation and $\mathcal{X} / P_{\mathcal{X}}$ is equicontinuous $([12,5.18])$. The flow $\mathcal{X}$ is then called proximally equicontinuous. In general, the smallest closed invariant equivalence relation $R$ on $\mathcal{X}$ such that $\mathcal{X} / R$ is almost periodic is called the equicontinuous structure relation of $\mathcal{X}$ and is denoted by $S_{\mathcal{X}}^{e}$. It is the smallest closed invariant equivalence relation on $X$ which contains $Q_{\mathcal{X}}([2$, p. 127]). Consequently, $\mathcal{X}$ is almost periodic if and only if $Q_{\mathcal{X}}=\Delta_{X}$. If the flow $\mathcal{X}$ is minimal, then $S_{\mathcal{X}}^{e}=Q_{\mathcal{X}}$ (this is also true for a large class of nonabelian flows) (114 Theorem 2.13]). We denote $\mathcal{X}^{e}:=\mathcal{X} / S_{\mathcal{X}}^{e}$.

If $\varphi: \mathcal{X} \rightarrow \mathcal{Y}$ is an extension, $R_{\varphi}=\left\{\left(x_{1}, x_{2}\right) \in X \times X \mid \varphi\left(x_{1}\right)=\varphi\left(x_{2}\right)\right\}$ is the unique closed invariant equivalence relation on $X$ such that $\mathcal{Y}$ is canonically isomorphic to $\mathcal{X} / R_{\varphi}$ and $\mathcal{Y}$ is almost periodic if and only if $Q_{\mathcal{X}} \subset R_{\varphi}$. The extension $\varphi$ is $\{$ proximal $\}\{$ regionally proximal $\}\{$ distal $\}$ if any $x, x^{\prime}$ from the same fiber are \{proximal $\}$ \{regionally proximal $\}$ distal or equal to each other $\}$.

Let $\mathcal{X}=\langle T, X\rangle$ be a flow and $S$ a syndetic subgroup of $T$. The set of orbit closures under $S$ is a partition of $X$ if and only if the set of orbit closures under $T$ is a partition of $X([19,2.24])$. In particular, if $\mathcal{X}$ is minimal, the set of orbit closures under $S$ is a partition of $X$.

Let $\mathcal{Y}=\langle S, Y\rangle$ be a flow. We denote by $\mathcal{O}_{S}$ the set $\{\overline{S y} \mid y \in Y\}$ of orbit closures of $\mathcal{Y}$ and by $R\left(\mathcal{O}_{S}\right)$ the relation on $Y$ defined by $\left(y_{1}, y_{2}\right) \in R\left(\mathcal{O}_{S}\right) \Leftrightarrow \overline{S y_{1}}=\overline{S y_{2}}$. If $\mathcal{Y}$ is compact and $\mathcal{O}_{S}$ forms a partition of $\mathcal{Y}$, then:

(i) for every open subset $V$ of $Y$, the saturation $\operatorname{sat}_{\mathcal{O}_{S}}(V)$ of $V$ with respect to the equivalence relation $R\left(\mathcal{O}_{S}\right)$ (i.e., the set $\left.\bigcup_{y \in V} \overline{S y}\right)$ is equal to $S . V$ ([19, 2.30(ii)]); in particular, $R\left(\mathcal{O}_{S}\right)$ is open;

(ii) if $R\left(\mathcal{O}_{S}\right)$ is closed, then for every $y \in Y$ and every neighborhood $O$ of $\overline{S . y}$, there exists a neighborhood $V$ of $y$ such that $S . V \subset O([19,2.31$ (III)]).

If $\mathcal{Y}$ is compact minimal, $R\left(\mathcal{O}_{S}\right)$ is an equivalence relation which is open and closed ([19, 2.32]).

\section{Eigenvalues of almost PeRiodic flows}

Let $\mathcal{X}=\langle T, X\rangle$ be a flow. The group of eigenvalues of $\mathcal{X}$, denoted by $E V(\mathcal{X})$, is the subgroup of $(\widehat{T})_{d}$ consisting of continuous characters $\chi \in \widehat{T}$ for which there exists a continuous function $f: X \rightarrow \mathbb{T}$ such that $f(t . x)=\chi(t) f(x)$ for all $t \in T$ and $x \in X$. The function $f$ is then an eigenfunction of $\mathcal{X}$ which corresponds to $\chi$ and $\chi$ is an eigenvalue of $\mathcal{X}$ (the eigenvalue which corresponds to $f$ ).

The bijective correspondence that we are now going to describe will be used several times in the rest of the paper.

3.1. Let $\mathcal{X}=\langle T, X, \pi\rangle$ be an almost periodic compact minimal flow. Fix any $e \in X$. Then there is a unique (abelian) group structure on the space $X$, compatible with its topology, such that $e$ is the identity element and the orbital map $\pi^{e}: T \rightarrow X$, $t \mapsto t e$, a (continuous) group homomorphism ([2, p. 105], [28, IV(3.42)]). Denote the group operation on $X$ by $*$. We have $t e * x=t x$ for all $t \in T, x \in X$.

Let $\eta \in \widehat{X}$ be a continuous character of $X$. Denote by $\gamma$ the character $\eta \circ \pi^{e}$ of $T$. Then $\eta$ is an eigenfunction of $\mathcal{X}$ corresponding to the eigenvalue $\gamma$. (Indeed, $\eta(t x)=\eta(t e * x)=\eta(t e) \eta(x)=\gamma(t) \eta(x)$. 
Conversely, let $\gamma \in \widehat{T}$ be an eigenvalue of $\mathcal{X}$. Let $f$ be an eigenfunction corresponding to $\gamma$ and let $c=\overline{f(e)} \in \mathbb{T}$. Then the function $\eta=c f$ is also an eigenfunction of $\mathcal{X}$ corresponding to $\gamma$, and it has the property $\eta(e)=1$. Putting $x=e$ in $\eta(t x)=\gamma(t) \eta(x)$ we get $\gamma=\eta \circ \pi^{e}$. Also, since $\eta\left(t^{\prime} e * t e\right)=\eta\left(t^{\prime}(t e)\right)=\eta\left(\left(t^{\prime}+t\right) e\right)=$ $\gamma\left(t^{\prime}+t\right)=\gamma\left(t^{\prime}\right) \gamma(t)=\eta\left(t^{\prime} e\right) \eta(t e)$, we have by continuity $\eta\left(x^{\prime} * x\right)=\eta\left(x^{\prime}\right) * \eta(x)$; i.e., $\eta \in \widehat{X}$. Moreover, since $\mathcal{X}$ is minimal, $\eta$ is the unique element of $\mathrm{C}(X)$ such that $\gamma=\eta \circ \pi^{e}$ and also the unique eigenfunction corresponding to $\gamma$ which is at the same time a continuous character of $X$.

So we conclude that, once $e \in X$ is fixed, there is a bijective correspondence (described above) $\omega_{e}=\omega: E V(\mathcal{X}) \rightarrow \widehat{X}$, given by $\gamma=\eta \circ \pi^{e} \mapsto \eta$, between the set of eigenvalues $\gamma$ of the flow $\mathcal{X}$ and the set of continuous characters $\eta$ of the topological group $X$. For each eigenvalue $\gamma$ of $\mathcal{X}, \eta$ is the unique corresponding eigenfunction which maps $e$ to 1 , and each continuous character $\eta$ of $X$ is an eigenfunction of $\mathcal{X}$ whose (unique) corresponding eigenvalue is $\gamma=\eta \circ \pi^{e}$. Moreover, the map $\omega$ is an isomorphism of (discrete) groups.

The following proposition generalizes the so-called "topological discrete spectrum theorem" which deals with $\mathbb{Z}$-flows on metrizable compact spaces. A more general version than this proposition can be deduced by adapting [25, 3.8] and/or by combining this proposition with an adaptation of [15, 4.5]. The version below is sufficient for the purposes of this paper.

Proposition 3.2. Let $\mathcal{X}=\langle T, X\rangle$ and $\mathcal{Y}=\langle T, Y\rangle$ be almost periodic compact minimal flows. The following are equivalent:

(i) $E V(\mathcal{X}) \supset E V(\mathcal{Y})$;

(ii) for every $x \in X$ and $y \in Y$ there is a (unique) morphism $\varphi: \mathcal{X} \rightarrow \mathcal{Y}$ such that $\varphi(x)=y$;

$\left(\mathrm{ii}^{\prime}\right)$ there is a morphism $\varphi: \mathcal{X} \rightarrow \mathcal{Y}$.

Proof. (i) $\Rightarrow$ (ii): Suppose (i) holds. We will use the statements and notation from Section 3.1. Let $X$ (resp. $\mathcal{Y}$ ) be equipped with the unique group structure compatible with its topology, such that $x$ (resp. $y$ ) is the identity element and the orbital map $\pi^{x}: T \rightarrow X$ (resp. $\pi^{y}: T \rightarrow Y$ ) a continuous group homomorphism. The map $\varphi^{\prime}: T x \rightarrow T y$, defined by $\varphi^{\prime}(t x)=t y$ for $t \in T$, is a group homomorphism. We show $\varphi^{\prime}$ is continuous (with respect to the induced topologies). Let $U \cap T y$ be a neighborhood of $y$ in $T y$, where $U=N\left(\chi_{1}, \ldots, \chi_{n} ; V_{\varepsilon}\right):=\left\{y^{\prime} \in Y: \chi_{i}\left(y^{\prime}\right) \in\right.$ $\left.V_{\varepsilon}, i=1, \ldots, n\right\}$ for some $\chi_{1}, \ldots, \chi_{n} \in \widehat{Y}$ and $V_{\varepsilon}=\{z \in \mathbb{T}:|z-1|<\varepsilon\}$. Let $\omega_{y}^{-1}\left(\chi_{i}\right)=\gamma_{i}(i=1, \ldots, n)$. Since $E V(\mathcal{X}) \supset E V(\mathcal{Y})$, the $\gamma_{i}$ are in $E V(\mathcal{X})$. Let $\omega_{x}\left(\gamma_{i}\right)=\delta_{i} \in \widehat{X}$ and let $O=N\left(\eta_{1}, \ldots, \eta_{n} ; V_{\varepsilon}\right):=\left\{x^{\prime} \in X: \eta_{i}\left(x^{\prime}\right) \in V_{\varepsilon}, i=\right.$ $1, \ldots, n\}$. Then $O$ is a neighborhood of $x$ in $X$. Also $\varphi^{\prime}(O \cap T x) \subset U \cap T y$. Thus $\varphi^{\prime}$ is a continuous group homomorphism on a dense subgroup of $X$. It then (uniquely) extends to a group homomorphism $\varphi: \mathcal{X} \rightarrow \mathcal{Y}$. This group homomorphism is in particular a morphism of flows.

(ii) $\Rightarrow\left(\right.$ ii $\left.^{\prime}\right)$ : clear.

$\left(\right.$ ii' $\left.^{\prime}\right) \Rightarrow(\mathrm{i})$ : Let $\chi \in E V(\mathcal{Y})$ and let $\eta$ be a corresponding eigenfunction. Then $\chi \in E V(\mathcal{X})$ with a corresponding eigenfunction $\eta \circ \varphi$.

Corollary 3.3. Let $\mathcal{X}=\langle T, X\rangle$ and $\mathcal{Y}=\langle T, Y\rangle$ be almost periodic compact minimal flows. The following are equivalent:

(i) $E V(\mathcal{X})=E V(\mathcal{Y})$; 
(ii) for every $x \in X$ and $y \in Y$ there is a (unique) isomorphism $\varphi: \mathcal{X} \rightarrow \mathcal{Y}$ such that $\varphi(x)=y$;

$\left(\mathrm{ii}^{\prime}\right) \mathcal{X}$ and $\mathcal{Y}$ are isomorphic.

Proof. (i) $\Rightarrow$ (ii): Assume (i). Let $x, y \in X$ and let $f: \mathcal{X} \rightarrow \mathcal{Y}$ be a morphism such that $f(x)=y$ and let $g: \mathcal{Y} \rightarrow \mathcal{X}$ be a morphism such that $g(y)=x$ (they exist by Proposition [3.2). Then $(g \circ f)(t x)=t x$ for all $t \in T$. Since $g \circ f$ is uniformly continuous on $T x$, it can be uniquely extended to a continuous map on $X$. Hence $g \circ f=\operatorname{Id}_{X}$. The rest is clear.

\section{Regularly almost Periodic, Regionally distal, LOCALly almost PERIODIC AND ALMOST AUTOMORPHIC POINTS; ALMOST $1-1$ EXTENSIONS}

Definition 4.1. Let $\mathcal{X}=\langle T, X\rangle$ be a flow. A point $x \in X$ is called regularly almost periodic if for every neighborhood $U$ of $x$ there exists a syndetic subgroup $S$ of $T$ such that $S . x \subset U$. A flow $\mathcal{X}$ is pointwise regularly almost periodic if every point $x \in X$ is regularly almost periodic. If $\left(X, \mathcal{U}_{X}\right)$ is a uniform space, $\mathcal{X}$ is uniformly regularly almost periodic if for every $\alpha \in \mathcal{U}_{X}$ there is a syndetic subgroup $S$ of $T$ such that $S . x \subset \alpha[x]$ for all $x \in X$.

4.2. (i) If $x$ is a regularly almost periodic point in $\mathcal{X}$, every point from T.x is regularly almost periodic in $\mathcal{X}$.

(ii) If $Y$ is an invariant subset of $X$ in the flow $\mathcal{X}$ and $y \in Y$, then $y$ is regularly almost periodic in $\mathcal{X}$ if and only if it is regularly almost periodic in the subflow $\mathcal{Y}=\langle T, Y\rangle$ of $\mathcal{X}$.

(iii) If $f: \mathcal{X} \rightarrow \mathcal{Y}$ is a morphism of flows and $x$ a regularly almost periodic point in $\mathcal{X}$, then $f(x)$ is a regularly almost periodic point in $\mathcal{Y}$.

Proposition 4.3 (after [19] 5.15]). Let $\mathcal{X}=\langle T, X\rangle$ be an almost periodic compact minimal flow. Suppose that there is a point $x \in X$ which is regularly almost periodic. Then $\mathcal{X}$ is uniformly regularly almost periodic.

Proof. Since $\mathcal{X}$ is minimal, it suffices to prove that the subflow on $T x$ of $\mathcal{X}$ is uniformly regularly almost periodic. Fix $\alpha \in \mathcal{U}_{X}$. We want to show that there exists a syndetic subgroup $S$ of $T$ such that for every $t \in T$, S.tx $\subset \alpha[t x]$. Since $\mathcal{X}$ is almost periodic, there exists a $\beta \in \mathcal{U}_{X}$ so that for every $t \in T$ and every $y \in X$, $t . \beta[y] \subset \alpha[t y]$. Since $x$ is regularly almost periodic, there exists a syndetic subgroup $S$ of $T$ such that $S . x \subset \beta[x]$. Now we have $S . t x \subset t . \beta[x] \subset \alpha[t x]$ for all $t \in T$.

Definition 4.4. Let $\mathcal{X}=\langle T, X\rangle$ be a flow. Two points $x, y \in X$ are called regionally distal (to each other) if they are not regionally proximal. A point $x \in X$ is called regionally distal if it is regionally distal to every point $y \in X \backslash\{x\}$, i.e., if $Q_{\mathcal{X}}[x]=\{x\}$.

If $x$ is a regularly almost periodic point in a compact minimal flow, it is easy to see that $x$ is a distal point $([19,10.08])$. We now prove a stronger statement.

Proposition 4.5. Let $\mathcal{X}=\langle T, X\rangle$ be a compact minimal flow. If $x \in X$ is regularly almost periodic, it is regionally distal.

Proof. In the proof we use some notions and facts from Section 2 Suppose $x$ is regularly almost periodic, but not regionally distal. Let $y \in X, y \neq x$, be such 
that $(x, y) \in Q_{\mathcal{X}}$. Let $U$ be a neighborhood of $x$ such that $y \notin \bar{U}$. There exists a syndetic subgroup $S$ of $T$ such that $S . x \subset U$. Then $\overline{S . x} \subset \bar{U}$ and so $y \notin \overline{S . x}$. Hence $\overline{S . x} \cap \overline{S . y}=\emptyset$ (since the orbit closures under $S$ form a partition of $X$ ). Since $X$ is compact, there are disjoint open neighborhoods $O_{1}$ of $\overline{S . x}$ and $O_{2}$ of $\overline{S . y}$. There exists an open neighborhood $V$ of $x$ such that $S . V \subset O_{1}$. Let $V^{\prime}$ be an open neighborhood of $x$ such that $\overline{V^{\prime}} \subset V$. We now consider saturations with respect to the equivalence relation $R\left(\mathcal{O}_{S}\right)$. Since $\operatorname{sat}(V)=S V$ and $\operatorname{sat}\left(V^{\prime}\right)=S V^{\prime}$, we have $S V^{\prime} \subset \operatorname{sat}\left(\overline{V^{\prime}}\right) \subset S V \subset O_{1}$. The relation $R\left(\mathcal{O}_{S}\right)$ is closed, so sat $\left(\overline{V^{\prime}}\right)$ is closed. Hence $\overline{S . V^{\prime}} \subset O_{1}$. We can similarly find an open neighborhood $W^{\prime}$ of $y$ so that $\overline{S . W^{\prime}} \subset O_{2}$.

Since $(x, y) \in Q_{\mathcal{X}}=Q_{\mathcal{X}_{S}}$, there exists a $z \in X$ so that for every neighborhood $U$ of $z$ there is an $s \in S$ such that $s \cdot V^{\prime} \cap U \neq \emptyset$ and $s . W^{\prime} \cap U \neq \emptyset$. This would imply $z \in \overline{S . V^{\prime}}$ and $z \in \overline{S . W^{\prime}}$. This is impossible since $O_{1}$ and $O_{2}$ are disjoint.

Definition 4.6. Let $\mathcal{X}=\langle T, X\rangle$ be a flow. A point $x \in X$ is called almost automorphic if the following condition holds: for every net $\left(t_{\gamma}\right)$ in $T$, if the limit $y=\lim t_{\gamma} x$ exists, then the $\operatorname{limit} \lim \left(-t_{\gamma}\right) y$ exists and $\lim \left(-t_{\gamma}\right) y=x$. The flow $\mathcal{X}$ is called pointwise almost automorphic if every point $x \in X$ is almost automorphic. The set of all almost automorphic points of $\mathcal{X}$ is denoted by $A A(\mathcal{X})$.

Definition 4.7. Let $\mathcal{X}=\langle T, X\rangle$ be a compact minimal flow, $x \in X$. Define the set $D[x]$ to be the set of all $y \in X$ such that there are nets $\left(t_{\lambda}\right),\left(s_{\lambda}\right)$ in $T$ with $t_{\lambda} x \rightarrow x, s_{\lambda} x \rightarrow x$ and $\left(t_{\lambda}-s_{\lambda}\right) x \rightarrow y$.

Also define the set $E[x]$ to be the set of all $y \in X$ such that there is a net $t_{\lambda}$ in $T$ and a point $z \in X$ with $t_{\lambda} x \rightarrow z,-t_{\lambda} z \rightarrow y$.

Remark 4.8. Note that:

(i) $x$ is almost automorphic if and only if $E[x]=\{x\}$;

(ii) $D[x]=\bigcap\{\overline{(D(x, V)-D(x, V)) x}: V \in \mathcal{N}(x)\}$, where $\mathcal{N}(x)$ is the set of all neighborhoods of $x$;

(iii) $P_{\mathcal{X}}[x] \subset D[x](24$, p. 290]).

Proposition 4.9 (27. Theorem 1.2]). Let $\mathcal{X}=\langle T, X\rangle$ be a compact minimal flow, $x \in X$. Then $\overline{E[x]}=D[x]$.

Lemma 4.10 (after [24, p. 290]). Let $\mathcal{X}=\langle T, X\rangle$ be a compact minimal flow, $x \in X$ and $U$ a neighborhood of $x$. Then:

(i) If $E[x]=\{x\}$, there is a neighborhood $V$ of $x$ such that $-D(x, V) \subset D(x, U)$. In particular, $D(x, U) \cap(-D(x, U))$ is syndetic.

(ii) If $D[x]=\{x\}$, there are neighborhoods $V$ and $W$ of $x$ such that

$$
\begin{gathered}
D(x, V)-D(x, V) \subset D(x, U), \\
D(x, W)+D(x, W) \subset D(x, U) .
\end{gathered}
$$

Proof. Follows from the points (1), (2) and (3) of the proof of [24, Theorem 2.4]. The proof uses the above Proposition 4.9 .

Definition 4.11. Let $\mathcal{X}=\langle T, X\rangle$ be a flow. A point $x \in X$ is called locally almost periodic if for every neighborhood $U$ of $x$ there is a neighborhood $V$ of $x$ and a syndetic subset $A$ of $T$ so that $A V \subset U$. The flow $\mathcal{X}$ is pointwise locally almost periodic if every point $x \in X$ is locally almost periodic. 
The next proposition is adapted from [28]. The proof there uses elements from [22] and [24] and consists of:

(i) $\Rightarrow$ (ii): by [28, $\mathrm{V}(7.1) 8]$;

(ii) $\Rightarrow$ (i): by [28, $\operatorname{IV}(6.12) 1]$;

(i) $\Leftrightarrow$ (iii) $\Leftrightarrow$ (iv): by [28, $\operatorname{IV}(6.12) 7]$. Alternatively, the reader can follow the steps indicated in our sketch of the proof.

Proposition 4.12 (after 22] and [24]). Let $\mathcal{X}=\langle T, X\rangle$ be a compact minimal flow, $x \in X$. The following are equivalent:

(i) $x$ is almost automorphic;

(ii) $x$ is regionally distal;

(iii) $x$ is distal and locally almost periodic;

(iv) $x$ is distal and $\mathcal{X}$ is proximally equicontinuous.

Proof. (ii) $\Rightarrow$ (i): Assume the contrary. Then there is a net $\left(t_{\lambda}\right)$ in $T$ with $t_{\lambda} x \rightarrow y$ and $z_{\lambda}:=-t_{\lambda} y \rightarrow z \neq x$. Then $t_{\lambda} z_{\lambda} \rightarrow y$ and $t_{\lambda} x \rightarrow y$. Hence $(x, z) \in Q_{\mathcal{X}}$, a contradiction.

(i) $\Rightarrow$ (iii): This is the statement of 24, Theorem 2.4]. The proof there is based on the above Proposition 4.9 Lemma 4.10 and Remark 4.8(iii). (The same implication, without distality of $x$, is proved in [14, Theorem 3.4]. A stronger statement (namely (i) $\Leftrightarrow($ iii)) is proved in [22, Theorem 2.3].)

(iii) $\Rightarrow$ (iv): follows from [13, Theorem 3].

(iv) $\Rightarrow$ (ii): $Q_{\mathcal{X}}[x]=P_{\mathcal{X}}[x]=\{x\}$.

Remark 4.13. By Proposition 4.12 if a compact minimal flow $\mathcal{X}$ has an almost automorphic point, then $S_{\mathcal{X}}^{e}=P_{\mathcal{X}}$ (see Section 2). This is not true in general, but it is remarkable that, as we have mentioned in Section $2 S_{\mathcal{X}}^{e}=Q_{\mathcal{X}}$ for all compact minimal abelian flows. Various proofs (working also for a large class of nonabelian flows) include for example [14, Theorem 2.13], [2, p. 130], 24, Corollary 3.9], [28, $\mathrm{V}(1.17)]$ and a recent elegant proof in [3, Corollary 21]. As it was mentioned in [14, p. 176], another proof can be deduced from [27].

Corollary 4.14. Let $\mathcal{X}=\langle T, X\rangle$ be a compact minimal flow. If $x \in X$ is regularly almost periodic, it is almost automorphic.

Proof. Follows from Proposition 4.5 and Proposition 4.12,

Definition 4.15. Let $\mathcal{X}=\langle T, X\rangle, \mathcal{Y}=\langle T, Y\rangle$ be compact flows. An extension $\varphi: \mathcal{X} \rightarrow \mathcal{Y}$ for which there exists a point $x \in X$ with dense orbit, such that $\varphi^{-1}(\varphi(x))=\{x\}$, is called an almost $1-1$ extension.

Note that then $\varphi^{-1}(\varphi(t x))=\{t x\}$ for all $t \in T$, which justifies the name. It is easy to check that every almost $1-1$ extension is proximal.

Proposition 4.16 (after [26, 3.4], 28, V(6.1)5] and [9, p. 228]). Let $\mathcal{X}=\langle T, X\rangle$ be a compact minimal flow. Let $q: \mathcal{X} \rightarrow \mathcal{X}^{e}$ be the canonical map. Then:

(a) The following are equivalent:

(i) $\mathcal{X}$ has an almost automorphic point;

(ii) $q: \mathcal{X} \rightarrow \mathcal{X}^{e}$ is an almost $1-1$ extension;

(iii) there is an almost $1-1$ extension $\varphi: \mathcal{X} \rightarrow \mathcal{Y}$ with $\mathcal{Y}$ almost periodic.

(b) $A A(\mathcal{X})=\left\{x \in X \mid q^{-1}(q(x))=\{x\}\right\}$.

(c) $\mathcal{X}$ is pointwise almost automorphic if and only if it is almost periodic. 
(d) Denote $q_{A A}:=\left.q\right|_{A A(\mathcal{X})}: A A(\mathcal{X}) \rightarrow q(A A(\mathcal{X})$ ) and for every $Z \subset X$ (resp. $\left.W \subset X^{e}\right), Z_{A A}:=Z \cap A A(\mathcal{X})$ (resp. $W_{A A}:=W \cap q(A A(\mathcal{X}))$ ). Assume that $X_{A A}$ (resp. $X_{A A}^{e}$ ) is given the topology induced from $X$ (resp. $\left.X^{e}\right)$. Then:

(i) for every $Z \subset X, \quad q_{A A}\left(Z_{A A}\right)=q(Z) \cap X_{A A}^{e}$;

(ii) $q_{A A}: X_{A A} \rightarrow X_{A A}^{e}$ is a homeomorphism.

Proof. (a) (i) $\Rightarrow$ (ii): Let $x \in X$ be an almost automorphic point. Then $q^{-1}(q(x))=$ $S_{\mathcal{X}}^{e}[x]$ is equal to $Q_{\mathcal{X}}[x]$ (Remark 4.13), which is equal to $\{x\}$ by Proposition 4.12

(ii) $\Rightarrow$ (iii): Clear.

(iii) $\Rightarrow\left(\right.$ i): Let $x \in X$ be a point with dense orbit such that $\varphi^{-1}(\varphi(x))=\{x\}$. Then, since $\mathcal{Y}$ is almost periodic, $Q_{\mathcal{X}} \subset R_{\varphi}$ (see Section 2). Hence, if $\left(x, x^{\prime}\right) \in Q_{\mathcal{X}}$, then $\varphi(x)=\varphi\left(x^{\prime}\right)$ and so $x=x^{\prime}$. Thus $Q_{\mathcal{X}}[x]=\{x\}$. By Proposition 4.12, $x$ is almost automorphic.

(b): Follows from the proof of (a) and Proposition 4.12.

(c): Follows from (b) ( $q$ becomes a bijection).

(d) (i): Let $Z \subset X$. We have $q_{A A}\left(Z_{A A}\right)=q(Z \cap A A(\mathcal{X})) \subset q(Z) \cap q(A A(\mathcal{X}))=$ $q(Z) \cap X_{A A}^{e}$. Conversely, suppose that $y \in X^{e}$ belongs to $q(Z) \cap q(A A(\mathcal{X}))$. Then there is an $x_{1} \in Z$ and an $x_{2} \in A A(\mathcal{X})$ such that $q\left(x_{1}\right)=q\left(x_{2}\right)=y$. But since $x_{2} \in A A(\mathcal{X}), y=q\left(x_{2}\right)$ has a one element fiber. So $x_{1}=x_{2}$. Hence $y \in q(Z \cap A A(\mathcal{X}))$.

(ii): $q_{A A}$ is continuous and bijective. We show that $q_{A A}$ is closed. Let $F_{A A}=$ $F \cap X_{A A}$ be a closed subset of $X_{A A}$ (where $F$ is a closed subset of $X$ ). By (i), $q_{A A}\left(F_{A A}\right)=q(F) \cap X_{A A}^{e}$. Hence, since $q$ is closed, $q_{A A}\left(F_{A A}\right)$ is closed in $X_{A A}^{e}$.

The next proposition is proved in [7 p. 151] for almost $1-1$ extensions. A more general statement and a simpler proof that we are giving, as well as the corollary that follows the proposition, were suggested by Joe Auslander.

Proposition 4.17. Let $\mathcal{X}=\langle T, X\rangle, \mathcal{Y}=\langle T, Y\rangle$ be compact minimal flows. Suppose that $h: \mathcal{X} \rightarrow \mathcal{Y}$ is a regionally proximal extension. Then $E V(\mathcal{X})=E V(\mathcal{Y})$.

Proof. Clearly $E V(\mathcal{Y}) \subset E V(\mathcal{X})$. Conversely, let $\chi \in E V(\mathcal{X})$ and let $f$ be an eigenfunction corresponding to $\chi$. In order to prove that $\chi \in E V(\mathcal{Y})$, it is enough to prove that $f$ is constant on the fibers of $h$. Let $\left(x, x^{\prime}\right) \in Q_{\mathcal{X}}$. Then (by [2] p. 133]) there are nets $\left(x_{\lambda}\right),\left(x_{\lambda}^{\prime}\right)$ in $X$ and $\left(t_{\lambda}\right)$ in $T$ such that $x_{\lambda} \rightarrow x, x_{\lambda}^{\prime} \rightarrow x^{\prime}$, $t_{\lambda} x_{\lambda} \rightarrow x$ and $t_{\lambda} x_{\lambda}^{\prime} \rightarrow x$. From $f\left(t_{\lambda} x_{\lambda}\right)=\chi\left(t_{\lambda}\right) f\left(x_{\lambda}\right)$ we get $\chi\left(t_{\lambda}\right) \rightarrow 1$. Now from $f\left(t_{\lambda} x_{\lambda}^{\prime}\right)=\chi\left(t_{\lambda}\right) f\left(x_{\lambda}^{\prime}\right)$ we get $f(x)=f\left(x^{\prime}\right)$. Hence, since $h$ is regionally proximal, $f$ is constant on the fibers of $h$.

Corollary 4.18. Let $\mathcal{X}$ be a compact minimal flow. Then $E V(\mathcal{X})=E V\left(\mathcal{X}^{e}\right)$. In particular, the maximal equicontinuous factor of $\mathcal{X}$ is (up to isomorphism) the unique almost periodic flow whose group of eigenvalues is equal to $E V(\mathcal{X})$.

Proof. We have $S_{\mathcal{X}}^{e}=Q_{\mathcal{X}}$ (see Remark 4.13). Hence $q: \mathcal{X} \rightarrow \mathcal{X}^{e}$ is a regionally proximal extension. By Proposition 4.17 $E V(\mathcal{X})=E V\left(\mathcal{X}^{e}\right)$. The second part of the statement follows from Corollary 3.3 .

Proposition 4.19 ([2, p. 142]). Let $h: \mathcal{X} \rightarrow \mathcal{Y}$ be a distal extension of compact minimal flows. Then $h$ is open and all fibers of $h$ have the same cardinality. 


\section{Characterizations of Regular almost Periodicity IN COMPACT MINIMAL FLOWS}

In this section we introduce the "eigenvalue condition" and use it to prove our characterization theorems.

Definition 5.1. We say that a flow $\mathcal{X}=\langle T, X\rangle$ satisfies the eigenvalue condition if for any finite set $F \subset E V(\mathcal{X})$, the intersection $\bigcap_{\chi \in F}$ ker $\chi$ is a syndetic subgroup of $T$.

Theorem 5.2. Let $\mathcal{X}=\langle T, X\rangle$ be a compact minimal flow. The following are equivalent:

(i) $\mathcal{X}$ is uniformly regularly almost periodic;

(ii) $\mathcal{X}$ is pointwise regularly almost periodic;

(iii) $\mathcal{X}$ is almost periodic and satisfies the eigenvalue condition.

Proof. (i) $\Leftrightarrow$ (ii): Suppose $\mathcal{X}$ is pointwise regularly almost periodic. Then by Proposition 4.16)(c) and Corollary 4.14, $\mathcal{X}$ is almost periodic. Now by Proposition 4.3, $\mathcal{X}$ is uniformly regularly almost periodic. The opposite direction is clear.

(ii) $\Leftrightarrow$ (iii): Since (i) $\Leftrightarrow$ (ii), we may assume that $\mathcal{X}$ is almost periodic and prove that $\mathcal{X}$ is pointwise regularly almost periodic if and only if it satisfies the eigenvalue condition. Fix an $x \in X$. By Proposition 4.3 it is enough to prove that $x$ is regularly almost periodic if and only if $\mathcal{X}$ satisfies the eigenvalue condition. We will use the statements and notation from Section 3.1. Let $X$ be equipped with the unique group structure compatible with its topology, such that $x$ is the identity element and the orbital map $\pi^{x}: T \rightarrow X$ a continuous group homomorphism.

Suppose $x$ is regularly almost periodic. Let $\gamma_{1}, \ldots, \gamma_{n} \in E V(\mathcal{X})$. Let $\chi_{i}=$ $\omega\left(\gamma_{i}\right) \in \widehat{X}(i=1, \ldots, n), 0<\varepsilon<\sqrt{2}$ and $U=N\left(\chi_{1}, \ldots, \chi_{n} ; V_{\varepsilon}\right):=\left\{x^{\prime} \in X:\right.$ $\left.\chi_{i}\left(x^{\prime}\right) \in V_{\varepsilon}, i=1, \ldots, n\right\}$, where $V_{\varepsilon}=\{z \in \mathbb{T}:|z-1|<\varepsilon\}$. $U$ is a (basic) neighborhood of $x$, hence there is a syndetic subgroup $S$ of $T$ such that $S x \subset U$. Then $\chi_{i}(S x) \subset V_{\varepsilon}$, hence $\gamma_{i}(S) \subset V_{\varepsilon}$ and so $\gamma_{i}(S)=\{1\}(i=1, \ldots, n)$. Thus $S \subset \bigcap_{i=1}^{n} \operatorname{ker}\left(\gamma_{i}\right)$. Hence $\mathcal{X}$ satisfies the eigenvalue condition.

Conversely, suppose that $\mathcal{X}$ satisfies the eigenvalue condition. Let $O$ be a neighborhood of $x$. Then there are $\chi_{1}, \ldots, \chi_{n} \in \widehat{X}$ and $\varepsilon>0$ such that $U=$ $N\left(\chi_{1}, \ldots, \chi_{n} ; V_{\varepsilon}\right) \subset O$. Let $S=\bigcap_{i=1}^{n} \operatorname{ker}\left(\gamma_{i}\right)$, where $\gamma_{i}=\omega^{-1}\left(\chi_{i}\right) \quad(i=1, \ldots, n)$. Then $S$ is a syndetic subgroup of $T$ and $S x \subset U$. Hence $x$ is regularly almost periodic.

Theorem 5.3. Let $\mathcal{X}=\langle T, X\rangle$ be a compact minimal flow and $x \in X$. The following are equivalent:

(i) $x$ is regularly almost periodic;

(ii) $x$ is almost automorphic and $\mathcal{X}$ satisfies the eigenvalue condition.

Proof. Suppose that $x$ is regularly almost periodic. Then by Corollary 4.14, $x$ is almost automorphic. By Corollary 4.18, $E V(\mathcal{X})=E V\left(\mathcal{X}^{e}\right)$. Thus it suffices to prove the eigenvalue condition for $\mathcal{X}^{e}$. Let $q: \mathcal{X} \rightarrow \mathcal{X}^{e}$ be the canonical map. Since $q(x)$ is regularly almost periodic in $\mathcal{X}^{e}$ and $\mathcal{X}^{e}$ is almost periodic, then by Proposition $4.3 \mathcal{X}^{e}$ is uniformly regularly almost periodic. Hence by Theorem 5.2 $\mathcal{X}^{e}$ satisfies the eigenvalue condition.

Conversely, suppose that (ii) holds. By Corollary 4.18, $E V(\mathcal{X})=E V\left(\mathcal{X}^{e}\right)$. Hence by Theorem [5.2, $\mathcal{X}^{e}$ is pointwise regularly almost periodic. Fix a neighborhood $U$ of $x$. Let $y=q(x) \in X_{A A}^{e}$. Using the notation and the statement 
of Proposition 4.16, if $V_{A A}:=q_{A A}\left(U_{A A}\right)$, there is a neighborhood $V$ of $y$ in $X^{e}$ such that $V_{A A}=V \cap X_{A A}^{e}$. Since $y$ is regularly almost periodic in $X^{e}$, there is a syndetic subgroup $S$ of $T$ such that $S y \subset V$. Note that $S x \subset X_{A A}$. Hence $S y=q_{A A}(S x) \subset X_{A A}^{e}$. So $S y \subset V_{A A}$. Hence $S x=q_{A A}^{-1}(S y) \subset U_{A A} \subset U$.

The following example illustrates that the almost periodicity condition in Theorem 5.2 (iii) cannot be weakened to distality.

Example 5.4. Let $\mathcal{X}=\langle\mathbb{R}, X\rangle$ be a uniformly regularly almost periodic flow. Let $\varphi: \mathbb{R} \times X \rightarrow \mathbb{R}$ be a continuous function which satisfies the condition

$$
\varphi(s+t, x)=\varphi(t, x)+\varphi(s, t x)
$$

for all $s, t \in \mathbb{R}, x \in X$. Define a flow $\mathcal{Y}=\langle\mathbb{R}, X \times \mathbb{T}\rangle$ by

$$
t .(x, z)=\left(t x, z e^{2 \pi i \varphi(t, x)}\right) .
$$

Then by [10, 2.1], if $\varphi$ is not uniformly continuous (see [10, 2.1] for a concrete example), $\mathcal{Y}$ is a distal compact minimal flow which is not almost periodic and $E V(\mathcal{X})=E V(\mathcal{Y})$. It follows from Theorem 5.2 that $\mathcal{Y}$ is an example of a distal compact minimal flow which satisfies the eigenvalue condition but is not uniformly regularly almost periodic.

\section{Applications: The CASE When the Phase spaCE is 0-Dimensional}

Lemma 6.1 ([20, 24.26]). Let $G$ be a compact abelian topological group. The following are equivalent:

(i) $G$ is 0-dimensional;

(ii) $\chi(G)$ is a finite subgroup of $\mathbb{T}$ for every character $\chi \in \widehat{G}$.

We now deduce the characterization theorem of Gottschalk.

Theorem 6.2 ([18, 1.13]). Let $\mathcal{X}=\langle T, X, \pi\rangle$ be a compact minimal flow with $X$ 0 -dimensional. Then $\mathcal{X}$ is uniformly regularly almost periodic if and only if it is almost periodic.

Proof. One direction is clear. Conversely, suppose $\mathcal{X}$ is almost periodic. Let $\chi_{1}$, $\ldots, \chi_{n}$ be some eigenvalues of $\mathcal{X}$. Fix a point $e \in X$. Let $X$ be equipped with the unique group structure compatible with its topology, such that $e$ is the identity element and the orbital map $\pi^{e}: T \rightarrow X$ a continuous group homomorphism. Then $\chi_{i}=\delta_{i} \circ \pi^{e}(i=1, \ldots, n)$, where $\delta_{i}$ are some characters of $X$ (see Section 3.1 ). Since $X$ is 0 -dimensional, then, by Lemma 6.1 each $\delta_{i}(X)$ is a finite subset of $\mathbb{T}$. Hence each $\chi_{i}(T)$ is a finite subset of $\mathbb{T}$; i.e., each $\operatorname{ker}\left(\chi_{i}\right)$ is of finite index in $T$. Then $\bigcap_{i=1}^{n}$ ker $\chi_{i}$ is also of finite index in $T$. Hence $\mathcal{X}$ satisfies the eigenvalue condition. Now by Theorem $5.2, \mathcal{X}$ is uniformly regularly almost periodic.

Remark 6.3. It is a theorem of R. Ellis ([11, Theorem 3]) that a distal compact minimal flow on a 0-dimensional phase space, whose acting group is generative, is pointwise regularly almost periodic. (A topological group is generative if it can be generated by a compact neighborhood of 0.) D. McMahon and T. S. Wu ([23, 3.11]) extend the class of acting groups for which Ellis' theorem remains true: $T$ can be a direct product of a compactly generated separable group and a compact group. (A topological group is compactly generated if it can be generated by a compact subset.) We believe that Ellis' theorem is true for a yet larger class of groups and that a condition on $T$ (under which Ellis' theorem still holds) can be 
formulated in terms of (discrete) syndetic subgroups of $T$ and/or characters of $T$. Some conditions on $T$ must be assumed, as was shown by an example in [23].

The next corollary shows that, in the context of 0-dimensional phase spaces, we can weaken both conditions in Proposition 4.3 and still get the same conclusion.

Corollary 6.4. Let $\mathcal{X}=\langle T, X\rangle$ be a distal compact minimal flow with $X 0$ dimensional. Suppose that there is a point $x \in X$ which is locally almost periodic. Then $\mathcal{X}$ is uniformly regularly almost periodic.

Proof. The point $x$ is distal and locally almost periodic. Hence, by Proposition 4.12 $\mathcal{X}$ is proximally equicontinuous. Also, since $Q_{\mathcal{X}}=S_{\mathcal{X}}^{e}$ (Remark 4.13) and $\mathcal{X}$ is distal, we have $S_{\mathcal{X}}^{e}=\Delta_{X}$, i.e., $\mathcal{X}$ is almost periodic. (This is proved in a different way in [17, Theorem 1].) Now by Theorem [6.2, $\mathcal{X}$ is uniformly regularly almost periodic.

Remark 6.5. If the distality condition in Corollary 6.4 is omitted, the statement is not true $(19,12.63]$ can be used as a counterexample).

6.6. Let $\mathcal{X}=\langle T, X\rangle$ be a compact minimal flow. We say that $\mathcal{X}$ is dynamically homogenous if for every two points $x, y \in X$ there is $\varphi \in \operatorname{Aut}(\mathcal{X})$ such that $\varphi(x)=y$. If $\mathcal{X}$ is almost periodic, it is dynamically homogenous (follows from Corollary 3.3). Conversely, if $\mathcal{X}$ is dynamically homogenous, it is distal ([28, IV(3.46)]).

Corollary 6.7. Let $\mathcal{X}=\langle T, X\rangle$ be a compact minimal flow on a metrizable 0dimensional phase space $X$. Then $\mathcal{X}$ is uniformly regularly almost periodic if and only if it is dynamically homogenous.

Proof. By Section [6.6] and [2, p. 45], a compact minimal flow $\mathcal{X}$ on a metrizable phase space is almost periodic if and only if it is dynamically homogenous. Hence the corollary follows from Theorem 6.2 .

In the next example we show that the metrizability condition in Corollary 6.7 cannot be omitted. So Ellis' theorem (mentioned in Remark 6.3) does not hold for an arbitrary acting group and an arbitrary 0-dimensional compact phase space even if the distality of the flow is replaced by a stronger condition of dynamical homogeneity. We start with an example from [23, p. 265], but develop it further in order to achieve dynamical homogeneity.

Example 6.8. Let $X=\prod_{-\infty}^{\infty} \mathbb{Z} / 2 \mathbb{Z}$ and $T=\bigoplus_{-\infty}^{\infty} \mathbb{Z} / 2 \mathbb{Z}$. $X$ is a compact metrizable 0-dimensional group, and $T$ is a dense subgroup of $X$. Define a flow $\mathcal{X}=\langle T, X\rangle$ by assuming that $T$ acts on $X$ via the group operation. $\mathcal{X}$ is then an almost periodic compact minimal flow. Let $e^{n} \in X(n \in \mathbb{Z})$ be the elements defined by

$$
e_{k}^{n}= \begin{cases}1, & \text { if } k=n \\ 0, & \text { if } k \neq n\end{cases}
$$

Let $\mathbf{0}$ (respectively $\mathbf{1}$ ) be the element of $X$ whose all coordinates are 0 (respectively $1)$. If $x \in X$, we denote $\bar{x}=\mathbf{1}+x$. Let $Y=X \times(\mathbb{Z} / 2 \mathbb{Z})$. We define a countable family $\left(\theta_{n}\right)_{n \in \mathbb{Z}}$ of homeomorphisms of $Y$, in the following way:

$$
\theta_{n}(x, z)=\left(\bar{x}+e^{n}, z+x_{n-1}+x_{n+1}\right) .
$$

We have $\theta_{n}^{2}=\operatorname{id}_{Y}, \theta_{m} \circ \theta_{n}=\theta_{n} \circ \theta_{m}$, for all $m, n \in \mathbb{Z}$. The group $\operatorname{Homeo}(Y)$, with the topology of pointwise convergence, naturally acts on $Y$. Let $H$ be a subgroup 
of this group, generated by the set of all $\theta_{n}$. Then the map $e_{n} \mapsto \theta_{n}$ from $T$ to $H$ extends to an isomorphism of topological groups. We define the action of $T$ on $Y$ by identifying $T$ and $H$. So if $t=e^{n_{1}}+\cdots+e^{n_{k}} \in T$, we put

$$
t(x, z)=\left(e^{n_{1}}+\cdots+e^{n_{k}}\right)(x, z)=\left(\theta_{n_{1}} \circ \cdots \circ \theta_{n_{k}}\right)(x, z) .
$$

With this action $\mathcal{Y}=\langle T, Y\rangle$ is a distal compact minimal flow on a metrizable 0 -dimensional phase space.

Define a map $p: \mathcal{Y} \rightarrow \mathcal{X}$ by $p(x, z)=x$. This map is a morphism of flows and so $\mathcal{Y}$ is a distal extension of $\mathcal{X}$. It is shown in [23, p. 266] that $((\mathbf{0}, z),(\mathbf{0}, z+1)) \in Q_{\mathcal{Y}}$. Hence $\Delta_{Y}=P_{\mathcal{Y}} \neq Q_{\mathcal{Y}}$. Since $Q_{\mathcal{Y}} \subset R_{p}$, it follows (see Section2) from Corollary 6.4 that

$$
S_{\mathcal{Y}}^{e}=Q_{\mathcal{Y}}=R_{p}=\{((x, z),(x, z+1)): x \in X\} .
$$

(So $\mathcal{X}$ is the maximal almost periodic factor of $\mathcal{Y}$.) In particular, by Proposition 4.12 and Corollary 4.14 none of the points of $\mathcal{Y}$ is regularly almost periodic. So we have a distal compact minimal flow on a 0-dimensional metrizable phase space none of whose points is regularly almost periodic. By Corollary 6.7, $\mathcal{Y}$ is not dynamically homogenous.

Now we reason as in [1, p. 609]. Let $E(\mathcal{Y})$ be the Ellis semigroup of $\mathcal{Y}$ (algebraically it is a group since $\mathcal{Y}$ is distal) and let $\mathcal{E}(\mathcal{Y})=\langle T, E(\mathcal{Y})\rangle$ be the canonical flow on it. Then (by [28, $\operatorname{IV}(3.9)]) \mathcal{E}(\mathcal{Y})$ is a distal compact minimal flow, which is not almost periodic (otherwise $\mathcal{Y}$ would be almost periodic as a homomorphic image of $\mathcal{E}(\mathcal{Y})$ ). Also since $\mathcal{E}(\mathcal{E}(\mathcal{Y})$ ) is canonically isomorphic to $\mathcal{E}(\mathcal{Y}), \mathcal{E}(\mathcal{Y})$ is dynamically homogeneous (by [1. Theorem 5] or [28, $\operatorname{IV}(3.46)]$ ). The phase space $E(\mathcal{Y})$ is 0 -dimensional as a subspace of the product $Y^{Y}$ of 0 -dimensional spaces. Thus (by Corollary 6.4) the flow $E(\mathcal{Y})$ is an example of a dynamically homogeneous compact minimal flow on a 0-dimensional phase space, none of whose points is regularly almost periodic. We conclude that the metrizability condition in Corollary 6.7 cannot be omitted (and that $E(\mathcal{Y})$ is not metrizable).

Corollary 6.9. Let $\mathcal{X}=\langle T, X\rangle$ be a distal compact minimal flow with $X$ 0-dimensional. Then the largest almost periodic factor of $\mathcal{X}$ is a uniformly regularly almost periodic flow on a 0 -dimensional phase space.

Proof. Consider the canonical map $q: \mathcal{X} \rightarrow \mathcal{X}^{e}$. Since $\mathcal{X}$ is distal, $q$ is open (by Proposition 4.19). So the phase space of $\mathcal{X}^{e}$ is 0 -dimensional. Hence, by Theorem 6.2, $\mathcal{X}^{e}$ is uniformly regularly almost periodic.

\section{Applications: A Characterization of Regularly almost periodic FUNCTIONS ON ARBITRARY TOPOLOGICAL GROUPS}

Consider the flow $\mathcal{X}=\left\langle T, B U C_{u}(T), \pi\right\rangle$, defined by $\pi(t, f)=f_{t}$ for any $t \in T$ and $f \in B U C(T)$, where $f_{t}(s)=f(s+t)$ for any $s \in T$. A function $f \in B U C(T)$ is called an almost periodic function on $T$ if it is an almost periodic point of the flow $\mathcal{X}$. The set of all almost periodic functions on $T$ is denoted by $A P(T)$. It forms a $C^{*}$-subalgebra of the $C^{*}$-algebra $B U C(T)$. For $f \in B U C(T)$ let

$$
X_{f}=\mathrm{Cl}_{B U C_{u}(T)}\left\{f_{t} \mid t \in T\right\} .
$$

If $f \in A P(T)$, the subflow $\mathcal{X}_{f}=\left\langle T, X_{f}\right\rangle$ of $\mathcal{X}$ is an almost periodic (compact) minimal flow. 
7.1. The Bohr compactification of a topological group $T$ is usually defined for LCA groups (see [20, 26.11]). In the approach that follows we work with arbitrary abelian topological groups. (We use [21, pp. 247-249] and the theory of Ellis semigroups ([2], [12, 28]).)

Let $A=A P(T)$ and consider the flow $\mathcal{A}=\langle T, A, \pi\rangle$, defined by $\pi(t, f)=f_{t}$ (a subflow of the above flow $\mathcal{X})$. Let $E(\mathcal{A})$ be the Ellis semigroup of this flow; i.e.,

$$
E(\mathcal{A})=\mathrm{Cl}_{\mathcal{F}_{p}(A, A)}\left\{\pi_{t} \mid t \in T\right\},
$$

where $\mathcal{F}_{p}(A, A)$ is the set of all maps from $A$ to $A$, equipped with the topology of pointwise convergence. Each member of $E(\mathcal{A})$ is an isometry of the $C^{*}$-algebra $A P(T)$ (which maps each orbit-closure $X_{f}$ onto itself). Since $\mathcal{X}$ is an equicontinuous flow with compact orbit-closures, then $E(\mathcal{A})$, equipped with composition, is a compact topological group. The convergence of nets in $E(\mathcal{A})$ is characterized by the following property: $\xi_{\sigma} \rightarrow \xi$ if and only if $\xi_{\sigma}(f)(t) \rightarrow \xi(f)(t)$ for all $f \in A$ and all $t \in T$.

Let $\alpha_{T}: T \rightarrow E(\mathcal{A})$ be the map $t \mapsto \pi_{t}$. It is a continuous group homomorphism. We call the pair $b T=\left(E(\mathcal{A}), \alpha_{T}\right)$ the Bohr compactification of $T$. (It can be shown that, in the case when $T$ is LCA, this notion coincides with the "standard" Bohr compactification.)

Now if $F \in C(b T)$, then $f=F \circ \alpha_{T} \in A P(T)$. Conversely, if $f \in A P(T)$, the function $F=\widetilde{f}$, defined by $\widetilde{f}(\xi)=\xi(f)(0)$, is continuous on $b T$ and $f=\widetilde{f} \circ \alpha_{T}$. The map $\theta: A P(T) \rightarrow C(b T)$, defined by $f \mapsto \widetilde{f}$, is an isometric isomorphism of $C^{*}$-algebras. The restriction $\theta:(\widehat{T})_{d} \rightarrow \widehat{b T}$ is an isomorphism of the groups of continuous characters of $T$ and $b T$.

We define an action of $T$ on $E(\mathcal{A})$ by $(t, \xi) \mapsto \pi_{t} \circ \xi$. The resulting flow $\mathcal{E}(\mathcal{A})=$ $\langle T, E(\mathcal{A})\rangle$ is almost periodic compact minimal and $E V(\mathcal{E}(\mathcal{A}))=(\widehat{T})_{d}$. For a fixed $f \in A P(T)$, the map $\mathrm{ev}_{f}: b T=E(\mathcal{A}) \rightarrow X_{f}$, defined by $\mathrm{ev}_{f}(\xi)=\xi(f)$, is a surjective continuous group homomorphism and a morphism of the flow $\mathcal{E}(\mathcal{A})$ onto the flow $\mathcal{X}_{f}$. The adjoint map $\widehat{\mathrm{ev}_{f}}: \widehat{X_{f}} \rightarrow \widehat{b T}$ is an injective group homomorphism. Denote $\Gamma_{f}=\widehat{\operatorname{evf}_{f}}\left(\widehat{X_{f}}\right)$. Then the maps $\omega: E V\left(\mathcal{X}_{f}\right) \rightarrow \widehat{X_{f}}, \quad \theta_{f}=\theta: E V\left(\mathcal{X}_{f}\right) \rightarrow \Gamma_{f}$ and $\widehat{\mathrm{ev}_{f}}: \widehat{X_{f}} \rightarrow \Gamma_{f}$ are group isomorphisms and $\theta_{f}=\widehat{\mathrm{ev}_{f}} \circ \omega$.

If we write $\chi^{\prime}=\omega(\chi)$ and $\tilde{\chi}=\theta(\chi)$ for $\chi \in E V\left(\mathcal{X}_{f}\right)$, the "eigenvalue relation" $\chi^{\prime}\left(\pi_{t}(f)\right)=\chi(t) \chi^{\prime}(f)=\tilde{\chi}\left(\pi_{t}\right)$ for every $t \in T$, extends to the relation $\chi^{\prime}(\xi(f))=$ $\tilde{\chi}(\xi)$ for every $\xi \in b T$.

Proposition $7.2([20,18.8$ and 18.9]). There exists a unique complex linear functional $M: A P(T) \rightarrow \mathbb{C}$ such that for every $f \in A P(T)$ and every $t \in T$ :

$$
\begin{aligned}
& M\left(f_{t}\right)=M(f) ; \\
& M(f)>0 \text { if } f \geq 0 \text { and } f \neq 0 ; \\
& M(1)=1 .
\end{aligned}
$$

Proof. Let $\lambda$ denote a normalized Haar measure on $b T$. Then $M_{b T}=\int_{b T} F d \lambda$ is a complex linear functional on $C(b T)$ which satisfies the conditions of the statement. Put $M=M_{b T} \circ \theta$. Then $M$ also satisfies the conditions. The uniqueness follows from [20, 18.9].

The mean value $M$ satisfies the following additional properties:

(i) for every $f \in A P(T),|M(f)| \leq M(|f|) \leq\|f\|$; 
(ii) for every $\chi_{1}, \chi_{2} \in \widehat{T}$,

$$
M\left(\chi_{1} \overline{\chi_{2}}\right)= \begin{cases}1, & \text { if } \chi_{1}=\chi_{2} \\ 0, & \text { if } \chi_{1} \neq \chi_{2}\end{cases}
$$

(iii) if $f_{n} \rightarrow f$ in $A P(T)$, then $M\left(f_{n}\right) \rightarrow M(f)$.

If $f \in A P(T)$, the map $a_{f}: \widehat{T} \rightarrow \mathbb{C}$, defined by $a_{f}(\chi)=M(f \bar{\chi})$, is called the Bohr transform of $f$. The Fourier characters of $f$ are the elements of the set

$$
F C(f)=\left\{\chi \in \widehat{T} \mid a_{f}(\chi) \neq 0\right\} .
$$

Proposition 7.3. Let $f \in A P(T)$. Then $f=0$ if and only if $F C(f)=\emptyset$. If $f \neq 0$, then for every $\varepsilon>0$ there exist $a_{1}, \ldots, a_{n}$ in $\mathbb{C}$ and $\gamma_{1}, \ldots, \gamma_{n}$ in $F C(f)$ such that $\left\|f-\sum_{i=1}^{n} a_{i} \gamma_{i}\right\| \leq \varepsilon$.

Proof. We use the notation from Section 7.1 and from the proof of Proposition 7.2. First notice that the statement holds for compact groups (29, Section 22 and 24] or [6, pp. 211-213]). Let $\widetilde{f}=\theta(f)$. Note that $\gamma \in F C(f)$ if and only if $\widetilde{\gamma}=\theta(\gamma) \in F C(\widetilde{f})$. Also for any $\varepsilon>0$, every " $\varepsilon$-approximation" $\sum_{i=1}^{n} a_{i} \delta_{i}$ of $\widetilde{f}$ is mapped by $\theta^{-1}$ to an " $\varepsilon$-approximation" $\sum_{i=1}^{n} a_{i} \gamma_{i}$ of $f$. So the statement for $f$ follows from the analogous statement for $\tilde{f}$ (which is true since $b T$ is compact).

Corollary 7.4. For any $f, g \in A P(T), f=g$ if and only if $a_{f}=a_{g}$.

Proof. If $a_{f}=a_{g}$, then $F C(f-g)=\emptyset$. Hence (by Proposition [7.3) $f=g$.

Lemma 7.5 ([20, 23.20]). Let $K$ be a compact topological group and let $\Gamma$ be a subgroup of $\widehat{K}$ such that for every $x \neq 0$ in $K$, there is a $\gamma \in \Gamma$ such that $\gamma(x) \neq 1$. Then $\Gamma=\widehat{K}$.

The following proposition (the frequency module theorem) is usually stated in the context of LCA acting groups (see [29, Section 35]). A variation of the statement and a different proof is given in 8 .

Proposition 7.6. If $f \in A P(T)$, the group $E V\left(\mathcal{X}_{f}\right)$ of eigenvalues of the flow $\mathcal{X}_{f}$ coincides with the subgroup of $\widehat{T}$ generated by $F C(f)$.

Proof. Let $\gamma \in F C(f)$. So $M(f \bar{\gamma}) \neq 0$. Then for every $t, s \in T, \pi^{f}(t)=\pi^{f}(s) \Rightarrow$ $f_{t}=f_{s} \Rightarrow M\left(f_{t} \bar{\gamma}\right)=M\left(f_{s} \bar{\gamma}\right) \Rightarrow \gamma(t) M(f \bar{\gamma})=\gamma(s) M(f \bar{\gamma}) \Rightarrow \gamma(t)=\gamma(s)$. Hence we can define a map $\delta^{\prime}:\left\{f_{t} \mid t \in T\right\} \rightarrow \mathbb{T}$ by $\delta^{\prime}\left(f_{t}\right)=\gamma(t)$ on the dense subgroup $\left\{f_{t} \mid t \in T\right\}$ of $X_{f}$. It is a continuous group homomorphism and so it uniquely extends to a continuous group homomorphism $\delta: X_{f} \rightarrow \mathbb{T}$. Since $\delta(t . f)=\gamma(t) \delta(f)$ for all $t \in T$, the map $\delta$ is an eigenfunction of $X_{f}$ whose corresponding eigenvalue is $\gamma$. Thus $\gamma \in E V\left(\mathcal{X}_{f}\right)$, i.e., $F C(f) \subset E V\left(\mathcal{X}_{f}\right)$.

Now by Lemma 7.5 and using the isomorphisms $\theta_{f}$, $\widehat{\mathrm{ev}_{f}}$ and $\omega$ (see Section 7.1), in order to prove that $F C(f)$ generates $E V\left(\mathcal{X}_{f}\right)$, it is enough to prove that for a $\xi \in b T$, if $\widetilde{\gamma}(\xi)=1$ for all $\gamma \in F C(f)$, then $\widetilde{\chi}(\xi)=1$ for all $\chi \in E V\left(\mathcal{X}_{f}\right)$. Let $\xi \in b T$ be such that the "if" part holds. Then $M(\xi(f) \bar{\gamma})=\widetilde{\gamma}(\xi) M(f \bar{\gamma})=M(f \bar{\gamma})$ for every $\gamma \in F C(f)$. Hence (by Corollary [7.4) $\xi(f)=f$. But now $\chi^{\prime}(f)=\chi^{\prime}(\xi(f))=$ $\widetilde{\chi}(\xi) \chi^{\prime}(f)$, hence $\tilde{\chi}(\xi)=1$, for all $\chi \in E V\left(\mathcal{X}_{f}\right)$. 
The next corollary is proved in [4] for $T=\mathbb{R}$ in a different way.

Corollary 7.7 ([4, Theorem IV.2]). Let $f, g \in A P(T)$. Then the flows $\mathcal{X}_{f}$ and $\mathcal{X}_{g}$ are isomorphic if and only if the groups generated by $F C(f)$ and $F C(g)$ are isomorphic.

Proof. Follows from Proposition 7.6 and Corollary 3.3

Corollary 7.8. Every almost periodic compact minimal flow $\mathcal{X}=\langle T, X\rangle$ having a countable group of eigenvalues is isomorphic to a flow $\mathcal{X}_{f}$ for some $f \in A P(T)$.

Proof. Let $E V(\mathcal{X})=\left\{\chi_{1}, \chi_{2}, \chi_{3}, \ldots\right\}$. Choose any nonzero complex numbers $a_{1}$, $a_{2}, a_{3}, \ldots$ so that $\sum_{i}\left|a_{i}\right|<\infty$ and define $f: T \rightarrow \mathbb{C}$ by $f(t)=\sum_{i} a_{i} \chi_{i}(t)$. Then $f \in A P(T)$ and $F C(f)=\left\{\chi_{1}, \chi_{2}, \chi_{3}, \ldots\right\}$. Hence, by Proposition 7.6, $E V(\mathcal{X})=$ $E V\left(\mathcal{X}_{f}\right)$. So by Corollary $3.3, \mathcal{X}$ and $\mathcal{X}_{f}$ are isomorphic.

Definition 7.9. Let $f \in B U C(T)$. Then $f$ is called periodic if there is a syndetic subgroup $S$ of $T$ such that $f(t+s)=f(t)$ for every $t \in T$ and $s \in S$. The function $f$ is called limit-periodic if it is a uniform limit of a sequence of periodic functions. The function $f$ is called regularly almost periodic if it is a regularly almost periodic element of the flow $\mathcal{X}=\left\langle T, B U C_{u}(T)\right\rangle$.

Theorem 7.10. For a function $f \in A P(T)$ the following are equivalent:

(i) $f$ is regularly almost periodic;

(ii) for every finite set $\left\{\chi_{1}, \ldots, \chi_{n}\right\} \subset F C(f)$, the intersection of the kernels $\bigcap_{i=1}^{n} \operatorname{ker}\left(\chi_{i}\right)$ is a syndetic subgroup of $T$;

(iii) $f$ is limit-periodic.

Proof. (i) $\Leftrightarrow$ (ii): It follows from Proposition 7.6 that condition (ii) is equivalent to the following condition: for every finite set $\left\{\chi_{1}, \ldots, \chi_{n}\right\} \subset E V\left(\mathcal{X}_{f}\right)$, the intersection of the kernels $\bigcap_{i=1}^{n} \operatorname{ker}\left(\chi_{i}\right)$ is a syndetic subgroup of $T$. Hence, by Theorem 5.2, we have (i) $\Leftrightarrow($ ii).

(ii) $\Rightarrow$ (iii): assume (ii) holds. Fix an $\varepsilon>0$. By Proposition 7.3, there is a trigonometric polynomial $g^{\varepsilon}$ such that $\left\|f-g^{\varepsilon}\right\|<\varepsilon$ and $F C\left(g^{\varepsilon}\right) \subset F C(f)$. Hence, by what we assumed, the intersection of the kernels of all characters that appear in $F C\left(g^{\varepsilon}\right)$ is a syndetic subgroup $S$ of $T$. So $g^{\varepsilon}$ is periodic.

(iii) $\Rightarrow(\mathrm{i})$ : Suppose $f$ is limit-periodic. Let $f=\lim _{n \rightarrow \infty} g^{(n)}$ in $B U C_{u}(T)$ and let, for each $n, S_{n}$ be the syndetic subgroup of periods of $g^{(n)}$. Fix an $\varepsilon>0$. Let $n$ be such that $\left\|f-g^{(n)}\right\|<\varepsilon / 2$. Then for every $s \in S_{n}$ we have

$$
\left\|f-f_{s}\right\| \leq\left\|f-g^{(n)}\right\|+\left\|g^{(n)}-g_{s}^{(n)}\right\|+\left\|g_{s}^{(n)}-f_{s}\right\|<\varepsilon / 2+0+\varepsilon / 2=\varepsilon .
$$

Hence $f$ is regularly almost periodic.

\section{Applications: The Case When the aCting group is Discrete}

8.1. When the acting group $T$ is discrete, the eigenvalue condition (Definition 5.1) for a compact minimal flow $\mathcal{X}=\langle T, X\rangle$ has a simpler form:

The kernel of any eigenvalue of $\mathcal{X}$ is of finite index in $T$. That is because the intersection of a finite number of subgroups of finite index is a subgroup of finite index.

We now deduce from Theorem 5.2 the characterization theorem of Gottschalk and Hedlund. 
Theorem 8.2 (19,5.31]). Let $\mathcal{X}=\langle T, X, \pi\rangle$ be a compact minimal flow with $T$ discrete. Then the following are equivalent:

(i) $\mathcal{X}$ is uniformly regularly almost periodic;

(ii) $\mathcal{X}$ is almost periodic and $X$ is 0 -dimensional.

Proof. (i) $\Rightarrow$ (ii): Suppose (i) holds. In particular $\mathcal{X}$ is almost periodic. Fix an element $e \in X$. Let $X$ be equipped with the unique group structure compatible with its topology, such that $e$ is the identity element and the orbital map $\pi^{e}: T \rightarrow X$ a continuous group homomorphism. Since, by Theorem $5.3, \mathcal{X}$ satisfies the eigenvalue condition, it follows from Sections 3.1 and 8.1 that for any character $\delta \in \widehat{X}, \delta(X)$ is a finite subgroup of $\mathbb{T}$. By Lemma $6.1 X$ is 0 -dimensional.

(ii) $\Rightarrow($ i): follows from Theorem 6.2

Corollary 8.3. Let $\mathcal{X}=\langle T, X\rangle$ be a compact minimal flow with $T$ discrete and $X$ metrizable. Then the following are equivalent:

(i) $\mathcal{X}$ is uniformly regularly almost periodic;

(ii) $\mathcal{X}$ is dynamically homogenous and $X$ is 0-dimensional.

Proof. By [2, p. 45], a compact minimal flow $\mathcal{X}$ on a metrizable phase space is almost periodic if and only if it is dynamically homogenous. Hence the corollary follows from Theorem 8.2

In Corollary 6.7 we had a condition (dynamical homogeneity) that is weaker than almost periodicity (but stronger than distality) which is equivalent to uniform regular almost periodicity in the context of 0 -dimensional metrizable phase spaces. In the next corollary we notice that, if in addition the acting group is $\mathbb{Z}$, there is a condition even weaker than distality, but still equivalent to uniform regular almost periodicity.

A compact $\mathbb{Z}$-flow $\mathcal{X}=\langle X, h\rangle$, with $(X, d)$ metric, is called weakly rigid if for every $\varepsilon>0$ and any $x_{1}, x_{2}, \ldots, x_{n}$ in $X$, there exists $k \in \mathbb{Z} \backslash\{0\}$ such that $d\left(h^{k}\left(x_{i}\right), x_{i}\right)<\varepsilon$ for all $i=1,2, \ldots, n$. Every distal compact (minimal) $\mathbb{Z}$-flow on a metrizable phase space is weakly rigid $([16,6.2])$.

Corollary 8.4. Let $\mathcal{X}$ be a compact minimal $\mathbb{Z}$-flow on a metrizable 0-dimensional space $X$. Then $\mathcal{X}$ is uniformly regularly almost periodic if and only if it is weakly rigid.

Proof. By [16, 6.7], in the context of this corollary, $\mathcal{X}$ is weakly rigid if and only if it is almost periodic. So the statement follows from Theorem 8.2 .

8.5. Let $\mathcal{X}$ be a $\mathbb{Z}$-flow. The subgroup of $\mathbb{R}$,

$$
\mathcal{M}(\mathcal{X})=\left\{\lambda \in \mathbb{R} \mid e^{2 \pi i \lambda(\cdot)} \in E V(\mathcal{X})\right\},
$$

is called the module of $\mathcal{X}$. It is isomorphic to the subgroup $E V(\mathcal{X})$ of $\widehat{\mathbb{Z}}$. The kernel of a character $\chi(n)=e^{2 \pi i \lambda n}$ of $\mathbb{Z}$ is of finite index if and only if $\lambda \in \mathbb{Q}$. So the eigenvalue condition for the $\mathbb{Z}$-flows has the form $\mathcal{M}(\mathcal{X}) \subset \mathbb{Q}$.

Example 8.6. The functions (=bisequences) $s$ defined by $s_{n}=\cos 2 \pi n+\sin \frac{n \pi}{3}$ and $s_{n}=\sum_{k=1}^{\infty} \frac{1}{k^{3}} e^{2 \pi i \frac{n}{k}}$ are regularly almost periodic, while the bisequence $r$ defined by $r_{n}=e^{i n}+e^{i \sqrt{2} n}$ is not. By Proposition 4.3, for each of the bisequences $s$, the associated flow $\mathcal{X}_{s}$ is uniformly regularly almost periodic. In particular, every bisequence from the orbit-closure $X_{s}$ is regularly almost periodic. On the other 
hand, none of the bisequences from $X_{r}$ is regularly almost periodic (although all of them are almost periodic).

Theorem 8.7. (a) There are (up to isomorphism) precisely $c=2^{\aleph_{0}}$ uniformly regularly almost periodic compact minimal $\mathbb{Z}$-flows, and the phase space of each of them is metrizable.

(b) A compact minimal $\mathbb{Z}$-flow has a regularly almost periodic point if and only if it is isomorphic to an almost $1-1$ extension of one of these flows.

(c) There exists a (unique) greatest uniformly regularly almost periodic compact minimal $\mathbb{Z}$-flow.

Proof. (a) We explicitly construct (up to isomorphism) all uniformly regularly almost periodic compact minimal $\mathbb{Z}$-flows. For every subgroup $\Lambda=\left\{\lambda_{1}, \lambda_{2}, \lambda_{3}, \ldots\right\}$ of $\mathbb{Q}$, we form a regularly almost periodic function $s(\Lambda)$ defined by $s(\Lambda)_{n}=$ $\sum_{k=1}^{\infty} \frac{1}{k^{2}} e^{2 \pi i \lambda_{k} n}$ and the corresponding uniformly regularly almost periodic flow $\mathcal{X}_{s(\Lambda)}=\left\langle X_{s(\Lambda)}, \sigma\right\rangle$. (Note that the action of $\mathbb{Z}$ on $X_{s(\Lambda)}$ by translations is in fact a shift-system. Here $\sigma$ denotes the shift $\sigma\left(s(\Lambda)_{n}\right)=s(\Lambda)_{n+1}$.) We have, by Proposition [7.6, $\mathcal{M}\left(\mathcal{X}_{s(\Lambda)}\right)=\Lambda$. Let $\Phi_{\mathbb{Z}}$ be the set of all flows we get in this way. Then (by Corollary (7.8) every uniformly regularly almost periodic compact minimal $\mathbb{Z}$-flow is isomorphic to some flow from $\Phi_{\mathbb{Z}}$. The cardinality of $\Phi_{\mathbb{Z}}$ is $c$ since there are $c$ subgroups of $\mathbb{Q}$. The phase space of each flow from $\Phi_{\mathbb{Z}}$ is metrizable, being a compact topological group with countably many continuous characters $([20,24.15])$.

(b) Follows from (the proof of) Theorem 5.3.

(c) By Section 8.5. Theorem [5.2 and Proposition 3.2 every uniformly regularly almost periodic compact minimal $\mathbb{Z}$-flow is a factor of the flow $\mathcal{X}_{s(Q)}$, i.e., this flow is a (unique) greatest uniformly regularly almost periodic compact minimal $\mathbb{Z}$-flow.

Example 8.8. An example of a compact minimal $\mathbb{Z}$-flow $\mathcal{H}$ on an (uncountable) metrizable 0-dimensional phase space $H$ with two points $\eta, \zeta \in H$ such that the set of regularly almost periodic points of $\mathcal{H}$ coincides with the complement of $\mathbb{Z} \eta \bigcup \mathbb{Z} \zeta$ in $H$ is given in [19, 12.55]. So $\mathcal{H}$ has a dense subset of regularly almost periodic points, but is not uniformly regularly almost periodic. (The flow $\mathcal{H}$ is a subflow of the symbolic flow on two symbols.)

\section{Applications: The Case When the acting group is $\mathbb{R}$}

Let $\mathcal{X}=\langle\mathbb{R}, X\rangle$ be a flow. The subgroup of $\mathbb{R}$,

$$
\mathcal{M}(\mathcal{X})=\left\{\lambda \in \mathbb{R} \mid e^{2 \pi i \lambda(\cdot)} \in E V(\mathcal{X})\right\},
$$

is called the module of $\mathcal{X}$. It is isomorphic to the subgroup $E V(\mathcal{X})$ of $\widehat{\mathbb{R}}$.

By [20, 18.15(a)], the complex linear functional on $A P(\mathbb{R})$, introduced in Proposition 7.2 has the form

$$
M(f)=\lim _{L \rightarrow \infty} \frac{1}{2 L} \int_{-L}^{L} f(x) d x .
$$

Let $f \in A P(\mathbb{R})$. The Fourier exponents of $f$ are the elements of the set

$$
F E(f)=\left\{\lambda \in \mathbb{R} \mid e^{2 \pi i \lambda(\cdot)} \in F C(f)\right\} .
$$

Thus

$$
F E(f)=\left\{\lambda \in \mathbb{R} \mid \lim _{L \rightarrow \infty} \frac{1}{2 L} \int_{-L}^{L} f(x) e^{-2 \pi i \lambda x} d x \neq 0\right\} .
$$


The module $\mathcal{M}\left(\mathcal{X}_{f}\right)\left(\mathcal{M}_{f}\right.$ for short) is called the module of $f$. We have $\mathcal{M}_{f}=$ $\left\{\lambda \in \mathbb{R} \mid e^{2 \pi i \lambda(\cdot)} \in E V\left(\mathcal{X}_{f}\right)\right\}$. By Proposition 7.6. $\mathcal{M}_{f}$ coincides with the subgroup of $\mathbb{R}$ generated by the Fourier exponents of $f$ and is isomorphic to the subgroup of $\widehat{\mathbb{R}}$ generated by the Fourier characters of $f$.

Thus in the case $T=\mathbb{R}$ we get the situation considered by Egawa in 9].

The following lemma is easy to prove.

Lemma 9.1. Let $\Lambda$ be a subset of $\mathbb{R} \backslash\{0\}$. Then the following are equivalent:

(i) $\mathbb{Z} \lambda_{1} \cap \cdots \cap \mathbb{Z} \lambda_{n} \neq\{0\}$ for any finite subset $\left\{\lambda_{1}, \ldots, \lambda_{n}\right\} \subset \Lambda$.

(ii) $\mathbb{Z} \lambda \cap \mathbb{Z} \lambda^{\prime} \neq\{0\}$ for any two elements $\lambda, \lambda^{\prime} \in \Lambda$.

(iii) $\Lambda \subset \mathbb{Q} r$ for some $r \in \mathbb{R}$.

9.2. Lemma 9.1 implies that, in the case when the acting group is $\mathbb{R}$, the eigenvalue condition has the form $\mathcal{M}(\mathcal{X}) \subset \mathbb{Q} r$ for some $r \in \mathbb{R}$.

Example 9.3. By Theorem 7.10 and Section 9.2 , the functions $f(x)=\cos 2 \pi x$, $f(x)=\sum_{n=1}^{\infty} \frac{1}{n^{2}} \sin \frac{x}{n}, f(x)=\sum_{n=1}^{\infty} \frac{1}{n^{2}} e^{i(n+1) \sqrt{2} x / n}$ are regularly almost periodic, while the functions $g(x)=e^{i x}+e^{i \sqrt{2} x}, \quad g(x)=\sum_{n=1}^{\infty} \frac{1}{n^{3}} e^{i \sqrt{n} x}$ are not. By Proposition 4.3. for each of the functions $f$, the associated flow $\mathcal{X}_{f}$ is uniformly regularly almost periodic. In particular, every function from the orbit-closure $X_{f}$ is regularly almost periodic. On the other hand, for each of the functions $g$, none of the functions from $X_{g}$ is regularly almost periodic (although all of them are almost periodic).

We now deduce from Theorem 5.3 and Theorem 5.2 the characterization theorems of Egawa.

Theorem 9.4 ([9, Theorem 1]). Let $\mathcal{X}=\langle\mathbb{R}, X\rangle$ be a compact minimal flow. Let $x \in X$. The following are equivalent:

(i) $x$ is regularly almost periodic;

(ii) $x$ is almost automorphic and $\mathcal{M}(\mathcal{X}) \subset \mathbb{Q} r$ for some $r \in \mathbb{R}$.

Proof. Follows from Section 9.2 and Theorem 5.3

Theorem 9.5 ([9, Theorem 2]). Let $\mathcal{X}=\langle\mathbb{R}, X\rangle$ be a compact minimal flow. The following are equivalent:

(i) $\mathcal{X}$ is pointwise regularly almost periodic;

(ii) $\mathcal{X}$ is almost periodic and $\mathcal{M}(\mathcal{X}) \subset \mathbb{Q} r$ for some $r \in \mathbb{R}$.

Proof. Follows from Section 9.2 and Theorem 5.2

9.6. It follows from Theorem 9.5 and Theorem 7.10 that a function $f \in A P(\mathbb{R})$ is limit-periodic if and only if $\mathcal{M}_{f} \subset \mathbb{Q} r$ for some $r \in \mathbb{R}$. For a direct proof see $[5$ I§6, Theorem 4].

In his statements of Theorem 9.4 and Theorem 9.5. Egawa assumed that the phase space is metrizable and essentially used that assumption in his proofs. Since our statements of Theorem 5.2 and Theorem 5.3 do not have that assumption, we are able to deduce metrizability and to give a classification of all uniformly regularly almost periodic compact minimal $\mathbb{R}$-flows as in the next theorem.

Theorem 9.7. (a) There are (up to isomorphism) precisely $c=2^{\aleph_{0}}$ uniformly regularly almost periodic compact minimal $\mathbb{R}$-flows and the phase space of each of them is metrizable. 
(b) A compact minimal $\mathbb{R}$-flow has a regularly almost periodic point if and only if it is isomorphic to an almost $1-1$ extension of one of these flows.

(c) There is no greatest uniformly regularly almost periodic compact minimal $\mathbb{R}$-flow. More precisely, there are (up to isomorphism) c mutually nonisomorphic uniformly regularly almost periodic compact minimal $\mathbb{R}$-flows such that every nontrivial uniformly regularly almost periodic compact minimal $\mathbb{R}$-flow is a factor of (precisely) one of these.

Proof. (a) We explicitly construct (up to isomorphism) all uniformly regularly almost periodic compact minimal $\mathbb{R}$-flows. For every subgroup $\Lambda=\left\{\lambda_{1}, \lambda_{2}, \lambda_{3}, \ldots\right\}$ of $\mathbb{R}$ contained in some $\mathbb{Q} r, r \in \mathbb{R}$, we form a regularly almost periodic function $f(\Lambda)$, defined by $f(\Lambda)(x)=\sum_{n=1}^{\infty} \frac{1}{n^{2}} e^{2 \pi i \lambda_{n} x}$ and the corresponding uniformly regularly almost periodic flow $\mathcal{X}_{f(\Lambda)}=\left\langle\mathbb{R}, X_{f(\Lambda)}\right\rangle$. We have, by Proposition 7.6 $\mathcal{M}\left(\mathcal{X}_{f(\Lambda)}\right)=\Lambda$. Let $\Phi_{\mathbb{R}}$ be the set of all flows we get in this way. Then (by Corollary[7.8) every uniformly regularly almost periodic compact minimal $\mathbb{R}$-flow is isomorphic to some flow from $\Phi_{\mathbb{R}}$. The cardinality of $\Phi_{\mathbb{R}}$ is $c$ since there are $c$ subgroups of $\mathbb{Q}$. The phase space of each flow from $\Phi_{\mathbb{R}}$ is metrizable, being a compact topological group with countably many continuous characters ([20, 24.15]).

(b) Follows from (the proof of) Theorem 5.3 .

(c) Each $\mathbb{Q} r$ is a one-dimensional vector subspace of the $\mathbb{Q}$-vector space $\mathbb{R}$, so there are $c$ subgroups $\mathbb{Q} r$ of $\mathbb{R}$ and the intersection of any two of them that are not equal to each other is $\{0\}$. Hence, by Proposition 3.2, for each two different subgroups $\mathbb{Q} r$, the only common factor of the corresponding flows $\mathcal{X}_{f(\mathbb{Q} r)}$ is the trivial flow. (In particular, they are mutually nonisomorphic.) It follows from this, Theorem 9.5 and Proposition 3.2 , that every nontrivial uniformly regularly almost periodic compact minimal $\mathbb{R}$-flow is a factor of one and only one of the flows $\mathcal{X}_{f(\mathbb{Q} r)}$, $r \in \mathbb{R}$.

Remark 9.8. The phase spaces of uniformly regularly almost periodic compact minimal flows are not necessarily metrizable for any acting group. The flow defined by the action of $b \mathbb{Z}$ on itself via the group operation is an example. Also the phase spaces of almost periodic compact minimal $\mathbb{R}$-flows are not necessarily metrizable.

\section{ACKNOWLEDGMENT}

The authors thank Joe Auslander for helpful comments and suggestions which improved the paper.

\section{REFERENCES}

[1] J. Auslander, Endomorphisms of minimal sets, Duke Math. J. 30 (1963), 605-614. MR 27:5245

[2] J. Auslander, Minimal flows and their extensions, North-Holland, Amsterdam, 1988. MR 89m:54050

[3] J. Auslander and M. Guerin, Regional proximality and the prolongation, Forum Math. 9 (1997), 761-774. MR 99f:54051

[4] J. Auslander and F. Hahn, Point transitive flows, algebras of functions and the Bebutov system, Fund. Math. 60 (1967), 117-137. MR 36:4541

[5] A. Besicovitch, Almost periodic functions, Cambridge University Press, Cambridge, 1932.

[6] C. Corduneanu, Almost periodic functions, Chelsea Publishing Company, New York, N.Y., 1989.

[7] J. Egawa, Eigenvalues of some almost automorphic functions, Kobe J. Math. 2 (1985), 149161. MR 87i:54085 
[8] J. Egawa, Eigenvalues of some almost periodic functions, Proc. Amer. Math. Soc. 115 (1992), 535-540. MR 92i:54041

[9] J. Egawa, A characterization of regularly almost periodic minimal flows, Proc. Japan Acad. 71 Ser. A (1995), 225-228. MR 96k:54063

[10] J. Egawa, Eigenvalues of some distal functions, Proc. Amer. Math. Soc. 126 (1998), 273-278. MR 98e:54038

[11] R. Ellis, Distal transformation groups, Pacif. J. Math. 8 (1958), 401-405. MR 21:96

[12] R. Ellis, Lectures on topological dynamics, W. A. Benjamin, Inc., New York, N.Y., 1969. MR 42:2463

[13] R. Ellis and W. Gottschalk, Homomorphisms of transformation groups, Trans. Amer. Math. Soc. 94 (1960), 258-271. MR 23:A960

[14] R. Ellis and H. Keynes, A characterization of the equicontinuous structure relation, Trans. Amer. Math. Soc. 161 (1971), 171-183. MR 43:8069

[15] A. Fink, Almost periodic differential equations, Springer-Verlag, Berlin, Heidelberg, New York, 1974. MR 57:792

[16] S. Glasner and D. Maon, Rigidity in topological dynamics, Ergod. Th. Dynam. Syst. 9 (1989), 309-320. MR 90h:54050

[17] W. Gottschalk, Characterizations of almost periodic transformation groups, Proc. Amer. Math. Soc. 7 (1956), 709-712. MR 18:141b

[18] W. Gottschalk, Substitution minimal sets, Trans. Amer. Math. Soc. 109 (1963), 467-491. MR 32:8325

[19] W. Gottschalk and G. Hedlund, Topological dynamics, Amer. Math. Soc. Colloq. Publ., vol. 36, Providence, R.I., 1955. MR 17:650e

[20] E. Hewitt and K. Ross, Abstract harmonic analysis, Springer-Verlag, Berlin, Heidelberg, New York, 1963.

[21] J. Kelley, General topology, D. Van Nostrand, Princeton, N.J., 1955. MR 16:1136c

[22] D. McMahon and T. S. Wu, On weak mixing and local almost periodicity, Duke Math. J. 39 (1972), 333-343. MR 49:1497

[23] D. McMahon and T.S. Wu, On the connectedness of homomorphisms in topological dynamics, Trans. Amer. Math. Soc. 217 (1976), 257-270. MR 54:1188

[24] D. McMahon and T. S. Wu, Notes on topological dynamics V: equicontinuous structure relations of minimal transformation groups, Bull. Inst. Math. Acad. Sinica 8 (1980), 283-294. MR 82b:54057b

[25] W. Shen and Y. Yi, Almost automorphic and almost periodic dynamics in skew-product semiflows. Memoirs Amer. Math. Soc., Vol 136, No 647, AMS, Providence, R.I., 1998. MR 99d:34088

[26] W. Veech, Almost automorphic functions on groups, Amer. J. Math. 87 Ser. A (1965), 719751. MR 32:4469

[27] W. Veech, The equicontinuous structure relation for minimal abelian transformation groups, Amer. J. Math. 90 (1968), 723-732. MR 38:702

[28] J. de Vries, Elements of topological dynamics. Mathematics and its Applications, 257. Kluwer Academic Publishers Group, Dordrecht, 1993. MR 94m:54098

[29] A. Weil, L' Integration dans les Groupes Topologiques. Hermann, Paris, 1940.

Department of Mathematics, University of Illinois at Urbana-Champaign, Urbana, ILLINOIS 61801

E-mail address: amiller@math.uiuc.edu

Department of Mathematics, University of Illinois at Urbana-Champaign, Urbana, ILLINOIS 61801

E-mail address: jrsnbltt@math.uiuc.edu 